\title{
LA DÉMOCRATIE AU XXIE SIÈCLE : LEURRE, MYTHE OU RÉALITÉ ?
}

\author{
Author(s) / Auteur(s) : \\ Andrée PIECQ \\ Master en Psychologie, \\ Directeur Scientifique, Formateur et Intervenant chez G.I.R.O.S. Institut Indépendant de Systémique des \\ Organisations \\ Secrétaire Générale de l'UES-EUS, Présidente d'honneur de S\&O \\ a.piecq@gmail.com
}

\begin{abstract}
Résumé :
Cet article est une tentative de soumettre la démocratie occidentale a une radiographie systémique à partir d'élément d'observation de la démocratie Athénienne jusqu'à la démocratie actuelle. Cette tentative part de l'analyse des contextes, des membres, des différents type de démocratie pour élaborer des hypothèses au niveau des règles mythiques et des mythes. La conclusion tentera de répondre à la question :la démocratie au XXIe siècle est elle un leure, un mythe ou une réalité?
\end{abstract}

Keywords / Mots-clés :

Démocratie, contextes, frontières, règles, mythes, incertitudes

\section{INTRODUCTION}

La Démocratie, «le gouvernement du peuple, par le peuple pour le peuple $»^{1}$ est d'après Winston Churchill le pire des régimes - à l'exception de tous les autres déjà essayés dans le passé. "Democracy is the worst form of government - except for all those other forms, that have been tried from time to time".

\section{LA DÉMOCRATIE, SES DIFFÉRENTES INSTANCES, SES LOIS TOUT SEMBLE PRENDRE L'EAU DEPUIS LA FIN DU XXÈME SIÈCLE. CERTAINS CONSIDÈRENT QUE C'EST LE RÉSULTAT :}

- d'un manque de maturité du citoyen ou d'une partie des citoyens,

- d'un dysfonctionnement de la représentativité,

- de la représentation comme forme de démocratie,

- $\quad$ de la démocratie elle-même en tant que régime.

Nous avons choisi de considéré, les difficultés rencontrées depuis la fin du 20 e siècle jusqu'à à ce jour par la Démocratie, comme l'expression d'un mal être des institutions démocratiques inhérent à son évolution. Cette position nous amène à nous interroger sur : " est-elle un leurre, un mythe, ou une réalité ?»

Pour tenter de répondre à cette question nous allons comparer à travers les siècles l'évolution de certains éléments qui en forment la structure depuis la Grèce antique à Athènes, en passant par la Démocratie américaine établie en 1828 .

Nous considérons dans cet article, «la Démocratie » comme un système, une organisation, inscrite dans des contextes et définie par sa structure. Notre méthodologie est celle reprise dans « De la pensée systémique à la pratique de l'organisation - le « Giroscope » ${ }^{2}$

\footnotetext{
${ }^{1}$ Définition qui provient des écrits de Périclès et qu'Abraham Lincoln (1809-1865).

${ }^{2}$ Andrée Piecq : De la pensée Systémique à la Pratique e l'Organisation - Le« giroscope » L'Harmattan 2011
} 
Pour accéder aux concepts étudiés nous utiliserons l'observation faites à partir de textes ${ }^{3}$.

\section{PLAN}

\section{La démocratie à Athènes}

1. Son contexte d'apparition

2. Ses membres : leurs rôles leurs statuts

3. Son type de démocratie et sa finalité

4. Ses règles

II. La démocratie au cours du Moyen Age en Europe (476-1492)

III. La démocratie en Angleterre (1215-1399)

IV. La démocratie en France (1789-1830)

V. Les Etats-Unis (1783-1791)

VI. La démocratie en Europe au XXIe siècle

VII. La démocratie au XXIe siècle est elle un leure, un mythe ou une réalité ?

VIII. Vivre en démocratie c'est accepter l'incertitude

\section{La démocratie Athénienne}

\section{$\underline{\text { Son contexte d'apparition }}$}

$\mathrm{Au} \mathrm{VI}$ e siècle av. J.-C., les différentes communautés Athéniennes sont confrontées à de graves problèmes :

L'inégalité politique dans la cité, les nobles ont le monopole du pouvoir politique.

Une situation à la fois financière et politique déclenche des luttes pour ce pouvoir.

Une nouvelle classe sociale urbaine riche (artisans, armateurs) veut pouvoir y participer et concurrence les nobles en achetant des armes et en participant à la guerre.

Une paupérisation rurale : les agriculteurs peu compétitifs face à la concurrence de la Grande-Grèce ${ }^{4}$ se vendent eux-mêmes pour payer leurs dettes et grossissent le nombre des pauvres et expriment leurs mécontentements.

Athènes est aux bords de l'explosion sociale.

En 594 avant J.C Solon ${ }^{5}$ est choisi comme arbitre par les différentes classes sociales, et effectue des réformes constitutionnelles.

$\mathrm{Au} \mathrm{V}$ siècle modification des lois : les pauvres peuvent participer à la gestion de la cité.

Ses membres : leurs statuts et leurs rôles.

Les citoyens de la ville

- Sont né de père Athénien - se battent pour défendre la ville - ont des propriétés foncières.

\footnotetext{
${ }^{3}$ Nous avons sélectionné des éléments historiques qui nous semblaient pertinents

${ }^{4}$ Régions côtières de l'Italie méridionale et de la Sicile.

${ }^{5}$ Il est considéré comme le père de la démocratie
} 
- Ont les pouvoirs politiques et judiciaires - ont droit de vote, sont éligible accèdent à la plus haute magistrature ;

- Font la guerre.

- Payent des impôts.

- Sont divisés en :

- Les hommes libres :

Les plus riches (les pentacosmédimnes) ont des terres, accèdent à toutes les magistratures dominent la vie politique athénienne (sont l'aristocratie), doivent financer les services publics, doivent également servir dans l'armé).

Les moins riches (les thètes) sont des paysans sans terre, sont exemptés d'impôts, sont_dispensés de servir dans l'armée, ont accès à l'Ecclésia ${ }^{6}$ et aux tribunaux.

- Les Esclaves :

Sont des biens et appartiennent à un maître, ne jouent aucun rôle.

- Les Femmes :

Sont considérées à vie comme des mineurs, ne jouent aucun rôle.

- Les Métèques (étrangers) :

Sont exclus - ne peuvent participer à la vie politique; s'ils ont défendus la cité et accomplis des exploits et payés ils peuvent devenir citoyen.

La gestion d'Athènes est faite par une Démocratie directe ${ }^{7}$ : « le peuple réuni en corps promulgue luimême les lois et prend lui-même les décisions le concernant.

Tous les citoyens décident de la politique par vote à main levé et à la majorité simple dans une assemblée, l'Ecclésia. Cette assemblée est toute-puissante et possède la souveraineté.

Sa finalité est l'égalité de tous les citoyens devant la loi et aussi la participation de tous à l'action communautaire.

\section{Le Moyen Age en Europe. (476-1492)}

Le Moyen Age est constitué de siècles guerriers, centrés sur les croisades. Le roi est absolu de droit divin et collabore avec l'église. La gestion directe des affaires du peuple ne l'intéresse pas.

Les rois et les nobles se préoccupent de la guerre : matériel, alliance, soldats.... En ce qui concerne les communautés villageoises ils se désintéressent de la manière dont elles se gèrent se contentant d'administrer la justice en laissant ainsi au peuple une grande autonomie.

Bien que du commerce, de l'artisanat et une forme d'industrie existent, la grande partie des ressources du peuple provient des produits de la terre qui sont considérés comme le bien de tous.

Dans certaines villes il y a des éléments de la démocratie qui apparaissent ainsi que dans certains villages. Dans ces villages les habitants se réunissaient pour discuter de tous les problèmes de l'administration: financiers, judiciaires et paroissiaux, de protection de la communauté contre les voleurs, les loups; du partage des récoltes. On retrouve aussi une administration démocratique dans des abbayes.

\footnotetext{
${ }^{6}$ Ecclésia ensemble des citoyens appelés à se réunir pour tirer au sort les magistrats parmi des volontaires de la classe la plus riche.

${ }^{7}$ La démocratie directe est un régie politique ou les citoyens exercent directement le pouvoir, sans aucun l'intermédiaire.
} 
La finalité profonde de ces tentatives de démocratie était de conserver la cohérence par la participation de tous à la gestion.

Ces essais de gestion peuvent être considérés comme une « Démocratie directe».

\section{La démocratie en Angleterre (1215-1688)}

1215 Les barons et les notables anglais s'opposent à l'absolutisme royal du roi Jean Sans Terre, totalement sans scrupule haït de tout son peuple (des paysans, de la noblesse, des bourgeois, jusqu'au clergé). Ils l'obligent à signer "La Grande Charte qui limite son pouvoir absolu, et l'empêche de lever des impôts extraordinaires qui devient la prérogative du Grand Conseil.

1240 Le terme Parlement anglais apparait.

1258-1265 tentatives d'Henri III à revenir en arrière et révolte des barrons.

1267 Le parlement restaure le pouvoir royal. Il sert d'arbitre entre le roi et les barons, il prend un rôle politique, n'a pas de texte législatif mais est un élément essentiel du pouvoir royal.

1272-1307 Edouard Ier consulte le parlement très souvent. Le Parlement consent l'impôt, des chevaliers élus, des grands barons des bourgeois participent aux jugements, présentent des pétitions.

1327-1377 Le parlement comprend 2 chambres : la Chambre des Lords et la Chambre des Communes Il fait pression pour qu'Edouard II abdique en faveur d'Edouard III. Les attributions judiciaires du Parlement augmentent. Il a un rôle politique de plus en plus grand.

1399 Le Parlement destitue Richard III le souverain ne peut plus aller à l'encontre du Parlement qui détient le pouvoir absolu.

1534 Henry VIII renforce le pouvoir royal, devient le chef de l'église.

1649 Olivier Cromwell installe une république dictatoriale et brutale.

1660 Les Stuarts accèdent à la royauté mais ont un pouvoir limité

1689 Charles II accepte de mettre fin aux arrestations arbitraires. Le Parlement met sur le trône Mary et Guillaume d'Orange qui signent " the Bill of Rights "qui garantit le droit à la liberté individuelle et qui donne le pouvoir au Parlement. Les membres du parlement sont librement élus, votent les lois et les garantissent, donnent leur consentement pour lever l'armée

L'Angleterre devient une Monarchie parlementaire ${ }^{8}$ et c'est le début de ce qui deviendra la Monarchie Constitutionnelle d'aujourd'hui avec seulement comme changement l'élection des députés au suffrage universel.

\section{La démocratie en France (1789-1830)}

Jusqu'à la prise de la Bastille la monarchie est absolutiste même, lorsque à l'apparition du siècle des lumières, l'économie et la société évoluent.

En 1748 Montesquieu fait paraitre son ouvrage majeur : "L'esprit des Lois ». Il y défend la thèse que si une assemblée ou un homme est dans une position sociale et ou politique dominante, un abus de pouvoir peut émerger. Il prône la séparation de 3 pouvoirs : l'exécutif, le législatif et le judiciaire. Il considère que la tyrannie s'installe si 2 pouvoirs sur trois sont détenus par une assemblée ou par un homme. Il souhaite qu'à chaque pouvoir un contre-pouvoir existe

\footnotetext{
${ }^{8}$ Dans une Monarchie parlementaire le roi détient le pouvoir avec un parlement qui détient le pouvoir législatif (faire des lois).
} 
Son système veut qu'à chaque pouvoir s'oppose un contre-pouvoir. Il ne fut guère écouté en France mais adopté par les USA naissants.

En 1762, Rousseau aura une grande influence avec « Le contrat social»

- Le pouvoir légitime doit être une convention, d'un pacte social ;

- Tout homme qui a souscrit au Pacte devient Citoyen de la Nation ;

- Tout citoyen vote directement ou indirectement la loi ;

- La loi n'est pas la somme des intérêts particuliers9 mais l'intérêt général.

- Ce qui marque les révolutionnaires c'est l'idée développée par Rousseau que l'homme s'est éloigné de la nature.

1789 Prise de la Bastille et fin du pouvoir absolu du roi.

1789-1791 Création de l'Assemblée Constituante. Elle abolit les privilèges, a un rôle législatif, rédige la constitution de la France, n'a pas de pouvoir exécutif, est élue par les citoyens payant un impôt. Les non catholiques peuvent y être élus. Les biens du clergé sont nationalisés.

1791 l'Assemblée législative est élue et établi la monarchie constitutionnelle ${ }^{10}$ la constitution est votée.

1792 La convention nationale proclame la république. L’Assemblée législative devient le parlement de la république. La royauté est déchue.

1793 La constitution républicaine est votée mais n'est jamais utilisée. Dictature de Robespierre, la Terreur s'installe et gouverne la France.

1794 Chute de Robespierre. Le comité de sureté générale est un organe d'exécution et est la police politique ; il coordonne l'effort de guerre.

1795 Le Directoire est à dominante bourgeoise et rétablit le suffrage censitaire, met en place le Conseil des Cinq-Cents, (assemblée législative qui propose les lois) et le Conseil des Anciens, (le Sénat) qui examine les lois proposées et les votes. Il subit de nombreux coups d'Etats

1799 Bonaparte arrête les parlementaires, dissout les Cinq-Cents.

1800 Une nouvelle constitution crée le Consulat, Bonaparte est nommé Premier Consul, il initiative les lois, les fait exécuter. Il nomme les ministres, consulte les Français par plébiscite, soumet l'église à l'Etat.

1802 Bonaparte est nommé consul à vie.

1804 Il met en place le Code Civil inspiré du droit romain, des lois de l'Ancien Régime et des apports de la Révolution. Les ouvriers sont étroitement surveillés, les opposants sont réduits au silence, la presse est censurée, tous les organes du pouvoir sont contrôlés et une dictature est établie. Il se proclame Empereur des Français.

1814 Chute de l'Empire et proclamation de la Première Restauration qui élabore « la Charte » qui est un compromis. Les acquis de la révolution sont conservés. La dynastie est rétablie. Le Roi est l'autorité, il gouverne par ordonnance, a le pouvoir législatif et judiciaire, peut dissoudre la chambre des députés et il modifie la majorité des pairs (nouvelles nominations). La liberté religieuse, l'impôt, l'accès aux emplois publics, liberté de la presse sont rétablis. Le catholicisme devient religion d'état.

1815 Les 100 jours de Napoléon et la défaite de Waterloo sont suivis par la Seconde Restauration qui essaye de recréer une unité La Charte n'est pas d'application et La Terreur Blanche s'installe.

1815-1820 Après la Terreur blanche la société est consolidée : recrutement militaires par tirage au sort, abolition de la censure, exprimer en public une opinion n'est plus criminel, ne sont punies que les

${ }^{9}$ L'importance du principe de totalité est déjà perçue par Rousseau.

${ }^{10}$ Dans monarchie constitutionnelle à cette époque les pouvoirs du roi (chef de l'état) sont limités par une constitution, des lois fondamentales ou la coutume. 
opinions transgressant la loi, le développement économique est favorisé, la croissance démographique augmente, la main d'œuvre rural est abondante. Des mesures protectionnistes contre l'Angleterre apparaissent. C'est le début de la révolution industrielle.

Février 1820, le duc de Berry est assassiné. Fin de la période libérale.

1820-1830 Mort de Louis XVIII. Clivage entre les libéraux et les ultras royalistes. Virage à droite, dissolution de la Chambre, renforcement politique et religieuse. Le Sacre de Charles X est adapté. Les mesures prises sont de plus en plus absolutistes, le roi fait des réponses inadaptées à l'opposition, les émigrés qui ont perdus leurs biens sont indemnisés, une loi est promulguée sur le sacrilège religieux. Les réponses inadaptées du souverain face à l'opposition entraînent la révolution de1830 et la mise en place de la monarchie de Juillet. La Nation retrouve sa souveraineté et un régime Parlementariste ${ }^{11}$ s'installe et en 1848 la Deuxième République est née.

\section{La démocratie aux Etats-Unis (1783-1897).}

1783 Le Traité de Paris met un terme à la guerre d'indépendance contre l'Angleterre et proclame unilatéralement l'indépendance et la création d'une fédération de 13 Etats autonomes qui repose sur trois principes fondamentaux : la République, la Démocratie et le fédéralisme.

1787 Publication de la constitution de ses 27 amendements (toujours d'actualité) qui instaure un gouvernement fédéral avec partage des compétences entre Etat fédéral (politique extérieure, défense, commerce) et Etats fédérés (justice, protection des droits individuels, l'instruction). Le pouvoir du peuple est souverain. Installation de la séparation des pouvoirs (influence de Montesquieu).

- Le pouvoir législatif : Chambre des représentants et le Sénat sont chargés de la rédaction des lois ;

- Le pouvoir exécutif est confié au président qui est en charge de l'exécution des lois, e qui est élus pour 4 ans.

- Le pouvoir judiciaire : indépendant, est supérieur aux deux autres car leurs actes peuvent lui être soumis. Il est confié à La Cour Suprême qui veille au respect de la Constitution dans les décisions législatives, exécutives et judiciaires et qui garantit les droits des individus.

Le président est le chef de l'Etat, du gouvernement, il est le commandant en chef de l'armée et des milices des États ; il nomme les ambassadeurs et conclut les traités. Il est élu pour 4 ans.

1789 George Washington est président. L'interprétation de la constitution donne naissance à deux écoles qui sont en opposition en ce qui concerne le partage des compétences entre Etat fédéral et fédérés. C'est ce qui donne naissance aux partis politiques américains.

1797 1er amendement «Le Congrès ne fera aucune loi relative à l'établissement d'une religion, ou à l'interdiction de son libre exercice ; ou pour limiter la liberté d'expression, de la presse ou le droit des citoyens de se réunir pacifiquement ou d'adresser au Gouvernement des pétitions pour obtenir réparations des torts subis. »

En 1823, Monroe édicte les principes de son administration. Les États-Unis s'interdisent de se mêler des affaires européennes.

1831-1865 La guerre de Sécession divise encore de nos jours. Plusieurs facteurs ont joué : les différences économiques, l'opposition sociale, l'incompréhension intellectuelle les progrès de

\footnotetext{
${ }^{11}$ Le régime parlementaire est le système constitutionnel ou les pouvoirs du cabinet ministériel et du Parlement sont en équilibre.
} 
l'abolitionnisme, le fanatisme et l'incompréhension. Elle est la première guerre moderne (matériel de guerre plus performant).

Ses conséquences, sont l'abolition de l'esclavage et lors de la reconstruction des Etats du Sud permettent aux Nordistes de prendre leur revanche. Apparition du Klu Klux Klan société secrète violente et raciste

1897 Victoire du capitalisme industriel sur l’Amérique agraire domination du parti républicaine.

L'Amérique est la première Démocratie moderne. C'est une démocratie présidentielle ${ }^{\mathbf{2}}$

Pour voter Il faut avoir la nationalité américaine, plus de 18 ans et disposer de ses droits civiques.

La justice est indépendante, des commissions parlementaires enquêtes si nécessaire sur les hommes politiques, la liberté de la presse et l'importance du droit.

Les minorités ont leurs libertés respectées. MAIS la lutte des Noirs américains pour l'obtention et la jouissance de leurs droits civiques continue. Ce n'est qu'en 1964 et 65 que l'égalité juridique entre blancs et noires devient réelle, cependant l'égalité économique et sociale n'est toujours pas acquise par la majorité de la population noire.

\section{La Démocratie en Europe au XXIe siècle}

Pour répondre à la question : «La démocratie au XXIe siècle leurre mythe ou réalité ? », ce chapitre étudie sur le plan systémique «les gouvernances ${ }^{13}$ démocratiques à régime représentatif de type parlementaire ${ }^{14}$ qui sont les plus nombreuses en Europe et même dans le monde ${ }^{15}$. C'est comme si la Démocratie était une VISION ${ }^{16}$ qui perdure à travers les siècles et dont le contexte, dans lequel elle s'inscrit, émerge à la suite de guerres, de difficultés sociales, pour limiter certains pouvoirs qu'il soit celui d'un roi ou d'une assemblée.

$\mathrm{Au}$ XXI siècle en Europe le contexte de la Vision « Démocratie » est différent.

C'est le contexte d'un monde où :

L'expansion démographique est galopante.

Le contexte climatique se modifie.

L'ère numérique apparait ainsi que l'universalisation de la communication par les réseaux sociaux.

Les intérêts commerciaux, économiques et financiers tiennent le haut du pavé.

La mondialisation (économique, culturel, politique,...) fait de plus en plus force de loi.

La création de la Communauté Européenne modifie les équilibres politiques.

\footnotetext{
${ }^{12}$ Régime présidentiel : est caractérisé par l'élection du Président au suffrage universel direct ou indirect. Aux Etats Unis il nomme, révoque et a autorité sur les ministres. L'exécutif relève exclusivement du président ; il est chef de l'État et chef du Gouvernement. Les assemblées ne peuvent mettre en cause sa responsabilité politique ne peut être mise en cause par, mais, mais il ne peut dissoudre les assemblées a seulement d'un droit de veto sur les textes législatifs.

${ }^{13}$ Les gouvernances démocratiques » sont des systèmes humains et ont de membres qui sont des hommes.

${ }^{14}$ Régime parlementaire est un régime politique $\mathrm{d}$ ou les pouvoirs sont séparés et collaborent. Les ministres sont responsables devant le Parlement. Le premier Ministre est à la tête du gouvernement (pouvoir exécutif) et du pouvoir législatif.

${ }^{15}$ Dans le monde : Albanie, Allemagne, Autriche, Bangladesh, Bosnie-Herzégovine, Cap Vert, Croatie, République tchèque, Dominique, Timor Oriental, Ethiopie, Finlande, Grèce, Hongrie, Islande, Inde ,Irak, Irlande, Israël, Italie, Lituanie, Liban, Macédoine, Malte, Maurice, Moldavie ,Monténégro, Népal, Pakistan, Pologne, Portugal, Samoa, Sain-Morin, Serbie, Singapour, Slovaquie, Slovénie, Trinité-et-Tobago, Tunisie, Turquie, Vanuatu, Le Royaume-Uni, Les Pays-Bas, La Belgique, La Bulgarie, Serbie, La Suède, Le Danemark, La Finlande,, Le Luxembourg, La France, L'Espagne, La Nouvelle Zélande, Le Zimbabwe.

${ }^{16}$ Andrée Piecq « De la Pensée Systémique à la Pratique de l'Organisation-Le « giroscope » p. 125 L'Harmattan
} 
Les interactions entre les pays occidentaux sont modifiées ce qui conduit à la paix entre les Etats mais en même temps les font participer à des conflits extérieurs. Depuis peu ils doivent se battre, sur leur propre territoire, contre le terrorisme pour défendre cette vision.

Notre hypothèse est que cette VISION et sa finalité ne peuvent sortir indemne de ce contexte.

Pour avoir une consistance opérationnelle (être organisée en fonction d'une cible à atteindre) ${ }^{17}$ c'est-àdire une finalité, cette vision (la démocratie) doit être déclinée en buts, objectifs, actions.

Nous faisons l'hypothèse que, depuis ses origines jusqu'à nos jours, la finalité de la Démocratie est «la souveraineté du peuple». Pour atteindre cette finalité 5 buts ${ }^{18}$ sont souhaités et nécessaires :

L'égalité de droit des citoyens.

La liberté de l'individu.

La protection de ses libertés et de ses droits.

Le pluralisme tolérant.

Les élections libres.

Ces buts aujourd'hui sont acceptés par tous, mais notre hypothèse est que c'est une acceptation purement idéologique et théorique. Des règles mythiques ${ }^{19}$ sont érigées en buts opérationnel à atteindre.

La description et l'observation des objectifs nécessaires à l'accomplissement de ces buts ainsi que celle des actions mises en œuvre pour les atteindre vont nous permettre de vérifier cette hypothèse.

3 objectifs permettent d'atteindre les buts de la démocratie représentative et de mettre en œuvre des actions nécessaires pour les atteindre.

Ces objectifs sont :

- L'établissement de la souveraineté du peuple

- La séparation des pouvoirs dans la démocratie représentative

- L'établissement des libertés fondamentales.

Les actions mises en œuvre pour atteindre ces objectifs sont

1. Pour établir la souveraineté du peuple,

- Créer une constitution, un acte fondamental qui régit et organise les rapports entre gouvernants et gouvernés et protège les citoyens.

L'observation montre que malgré cela la souveraineté du peuple est menacée et que des usurpations du pouvoir sont possibles.

- Créer des assemblées pour rassembler le peuple.

L'observation montre que cela ne peut fonctionner que si les finalités individuelles des assemblées sont orientées vers une finalité globale identique.

- Favoriser la volonté du citoyen à travers des assemblées restreintes qui les représentent. L'observation montre que tous les citoyens ne sont pas représentés toujours de la même façon.

- Toucher la totalité des nations (qui ont des formes différentes) en espérant une émergence universelle de la démocratie.

L'observation montre que ce qui va émerger est différent d'une souveraineté commune qui amalgamerait les nations ${ }^{20}$.

\footnotetext{
${ }^{17}$ Idem p. 124

${ }^{18}$ La finalité se décline en buts, objectifs, actions.

${ }^{19}$ Andrée Piecq « De la Pensée Systémique à la Pratique de l'Organisation-Le « giroscope » p. 137 L’Harmattan
} 
- Créer un régime représentatif de type parlementaire.

L'observation montre que des abus de pouvoir ou des déviations peuvent apparaître lorsque le droit de dissolution des chambres est discrétionnaire.

- Garantir l'intérêt général et la protection de l'intérêt individuel.

L'observation montre que ces intérêts peuvent être contradictoires et même paradoxaux, (ceux des élus ne correspond pas toujours à ceux des électeurs); les mandats sont cumulés par les élus; des lobbies financiers deviennent trop puissants.

- Mettre en place le suffrage universel libre avec l'obligation de vote après la présentation des programmes politiques.

L'observation montre qu'il ne suffit pas, « une tyrannie » de la majoritée ${ }^{11}$ peut apparaître ; dans certains pays dits «démocratiques» les élections se font sur haute surveillance et le scrutin n'est ni secrets ni libre.

- Créer des intermédiaires les partis (y compris ceux de l'opposition) des intermédiaires entre le peuple et le gouvernement pour permettre l'expression libre de toutes les opinions, et avoir des alternatives.

L'observation montre que les partis tentent d'obtenir des mandats politiques pour avoir le pouvoir et favorise les électeurs appartenant à leur «famille»; il y a un taux grandissant d'absentions lors des élections.

- Création du pouvoir législatif (le parlement), du corps exécutif (le gouvernement) du pouvoir judiciaire (les juges) indépendants l'un de l'autre.

L'observation montre que les dépositaires du pouvoir ne sont pas tous élus mais peuvent être nommé par les partis.

2. La séparation des pouvoirs dans la démocratie représentative

- Séparer les 3 pouvoirs législatifs exécutif et judiciaire

L'observation montre qu'il y a interdépendances et actions réciproques; dans l'Union Européenne 3 institutions La Commission européenne, le Conseil de l'Union européenne, le Parlement européen collaborent pour élaborer les règlements et les directives.

- Créer de nouvelles institutions internationales au-dessus des institutions nationales et qu'elle soit acceptées par tous.

L'observation montre que cela n'est possible que si une identité européenne et même mondiale existait, ce qui n'est pas le cas.

3. Les libertés fondamentales

Les actions mises en œuvre

- Assurer les droits primordiaux: le droit inaltérable, les droits de l'homme, les libertés publiques.

L'observation montre qu'il existe des nations qui lors de conflit internes ne respectes pas les droits e l'homme. Des nominations à des postes publics sont parfois arbitraires

\footnotetext{
${ }^{20}$ Le principe directeur de la Totalité : Toute organisation doit être considérée dans sa totalité, dans sa complexité et dans sa dynamique propre.

${ }^{21}$ Exemple : au Kosovo l'oppression de la minorité albanaise
} 
- Gérer pacifiquement les tensions et les conflits naissant du contraste entre les différentes composantes de la société et leurs diverses volontés.

L'observation montre que peuple à la possibilité de manifester son désaccord et de forcer ou d'empêcher une décision par «l'arme du nombre » ou par la désobéissance civique.

- Créer la charte des droits fondamentaux de l'Union européenne.

L'observation a montré sa difficulté d'exécution.

- Modifier le partage des richesses pour permettre le développement et la croissance de tous. L'observation montre qu'il risque d'avoir un effet vase communicant qui inverserait la procession des richesses sans amener l'équilibre.

- Maintenir la qualité de l'environnement.

L'observation montre que si chacun acceptait de faire des sacrifices une amélioration serait possible.

A partir de cette lecture non exhaustive de la finalité de La «démocratie parlementaire», des hypothèses émergent. Nous les déclinons en termes de principes directeurs car si des changements s'avèreraient possible c'est sur eux qu'ils devraient porter ${ }^{22}$.

Hypothèses au niveau des règles phénoménologiques implicites ${ }^{23}$ et des principes directeurs concernés :

C'est comme si une minorité à le pouvoir et agit indépendamment des électeurs : règles concernant le rôle et la fonction des élus, qui interrogent au niveau de la communication (indirecte) des frontières (perméables) des rétroactions (positives orientée changement en opposition à des rétroactions négatives (orientée homéostasie).

C'est comme si la diversité des électeurs n'étaient pas représentée (règles concernant les statuts des sous-systèmes et de membres touchant à la représentativité).

C'est comme si des risques de corruption pouvait apparaître (règles qui touchent à la fois à la fonction au rôle et aux actions des élus).

C'est comme si les actions faites par des élus visaient leur réélection (règles qui font fi de la garantie de protection à la fois des biens individuels et collectifs et qui touchent la fonction, les rôles et les actions.

C'est comme si les procédures mettaient des frontières entre élus et électeurs (règles touchant la communication)

C'est comme si il y avait des paradoxes entre avoir une opinion commune (règle de la souveraineté du peuple) et la liberté de l'opinion individuelle (libertés fondamentales) (règles touchant la communication)

C'est comme si il y avait des difficultés de communication entre élu/ électeurs (règles de la communication avec tous les pièges que cela peut comporter)

\section{La démocratie au XXIe siècle est elle un leurre, un mythe ou une réalité ?}

La définition généralement admise de la notion de leurre est: «dispositif destiné à tromper, généralement des animaux ».

\footnotetext{
${ }^{22}$ Andrée Piecq « De la Pensée Systémique à la Pratique de l’Organisation-Le « giroscope » p. 196 L'Harmattan

${ }^{23}$ Idem p. 135 
Si la vision démocratique était un leurre elle serait destinée à tromper les citoyens. Des dérives existent nous en avons relevées quelques-unes dans le chapitre précédent. Cependant toutes gouvernances démocratiques issues de cette vision admettent que la société est divisée en soussystème dont le statut, les rôles, les intérêts, les objectifs et les valeurs sont différents allant jusqu'à l'antagonisme. Un de de leurs objectifs « les libertés fondamentales » inscrites dans la Constitution est le garant de la gestion des tensions et des conflits qui peuvent émerger du contraste existant entre les différentes composantes de la société. Cette garantie permet, par la concertation sociale, le droit de grèves et de manifestation, de forcer ou d'empêcher de prendre une décision imminente, parfois non prévues, non acceptée ou souhaitée.

L'histoire nous apprend que la Démocratie a émergé après des conflits et que de nombreux changements de contextes en ont modifié la pratique jusqu'à nos jours et ce de façon cyclique.

La pratique de la démocratie N'EST PAS LA DEMOCRATIE qui est une idée, un concept, une vision mais pas une pratique, pas un ensemble d'actions qui tendent vers un but à atteindre, but qui peut se situer dans le court terme, le moyen terme ou le long terme. La pratique de la démocratie est aux mains des gouvernances démocratiques.

Parler de LA démocratie au lieu de "gouvernances démocratiques » est une confusion sémantique, une erreur de cible, une confusion de niveau logique ${ }^{24}$ dès lors toutes les analyses critiques, même les moins étoffées, les plus partisanes peuvent lui être faites.

Une «gouvernance démocratique » est gérée par des hommes et est considérée comme un système humain. Tout système humain a une vision de ce qu'il est ou de ce qu'il devrait être : un mythe. Il obéit à des règles mythiques qui engendrent des règles phénoménologiques qui s'observent à travers les buts effectuées pour atteindre les objectifs. Objectifs qui peuvent ne jamais être atteint dans le moyen terme ni même parfois dans le long terme car eux-mêmes relèvent du mythe.

Le mythe de la "gouvernance démocratique » est LA DEMOCRATIE et les règles mythiques qui en découlent sont la liberté de l'individu, la protection de ses libertés et de ses droits.

Les règles phénoménologiques observées semblent être parfois en contradiction systémique avec les règles mythiques (valoriser l'opinion commune et valoriser la liberté individuelle) dont elles sont issues.

La démocratie est la vision d'une "gestion de la cité », elle est un mythe, peut-être le plus beau des mythes de gestion de la cité : celui d'une cité gérée par des citoyens libres et égaux.

Revenons au quotidien, à la réalité. Notre réalité est la gestion, de l'Europe et du Monde, par des gouvernements dit démocratiques, des systèmes humains démocratiques qui n'ont pas nécessairement les mêmes finalités, et même si ils les ont, tentent de les atteindre avec des objectifs différents et des actions parfois contradictoires même paradoxales. En effet, ce qui peut donner l'illusion d'une direction partagée dans nos démocraties est parfois la simple rencontre d'opinions qui n'est pas pour autant une finalité commune, ni une action.

Tous les systèmes sont composés de membres, et les membres qui constituent la démocratie sont des hommes. Vouloir réduire un système à ses composants c'est faire fi du principe de totalité. Faire l'impasse sur l'unicité individuelle des membres ${ }^{25}$ peut être également porteurs d'erreurs : les « comportements » des éléments dépendent de ce qu'ils sont. Cette unicité individuelle des membres nous la considérons comme un des éléments des contextes du système.

Alors qu'elle issue proposer?

L'observation nous montre que lorsque des crises surviennent (inondations, attentas, catastrophes en tout genre) le citoyen reprend ses droits. En s'inscrivant dans l'action communautaire, le rapport aux élus se fluidifie, le mythe reprend force, reprend de la vigueur se ranime. C'est éphémère mais n'esse

\footnotetext{
${ }^{24}$ Andrée Piecq « De la Pensée Systémique à la Pratique de l'Organisation-Le « giroscope » p. 94-95 L'Harmattan

${ }^{25}$ Nous appelons unicité individuelle tous les éléments qui font la spécificité de chaque membre. Ici des hommes. Ces éléments font partie de leurs contextes d'appartenance (la culture, l'appartenance ethnique, etc...et de leur valeurs).
} 
pas l'indice de modification de comportements qui pourraient donner une vie nouvelle à la gouvernance démocratique?

\section{Vivre en démocratie c'est accepter l'incertitude.}

Vivre en étant gouverné par la vision démocratique en 2016, est difficile. Que ce soit sur le plan national ou international. C'est comme si cette valeur est bafouée, comme si son application dans le contexte actuel ne pouvait qu'engendrer des souffrances, c'est comme si la liberté individuelle, de la personne et dans certain cas du gouvernement, était devenue prépondérante. Partout nous entendons des cris provenant de volontés de séparation (le Brexit), de difficultés de trouver ses marques dans le fédéralisme (Flandre Wallonie et Bruxelles); des nominations ou des licenciements arbitraires, des crises financières, des problèmes d'extrémismes religieux (Daesh et les attentas),..

La certitude de vivre dans une société sans guerre qui accepte, de nouveaux équilibres politiques et religieux; de vivre dans une société prospère en pouvant, utiliser sans compter, les réserves naturelles n'existe plus. Cette perte de certitudes, de repères font émerger chez les citoyens des sentiments de doute, de peur du lendemain et provoque des remous au sein des gouvernements démocratiques. Certains médias et hommes politiques mette en cause «la gouvernance démocratique ». Certains réclament un régime fort dans lequel il n'y a ni liberté individuelle ni même collective. Dans une société démocratique, la souveraineté de la nation: l'égalité du droit des citoyens, la liberté de l'individu, les protections des libertés et des droits, le pluralisme, le suffrage universel, la liberté d'expression, la création des partis, le recours à la règle de la majorité, portent en eux l'incertitude du résultat. Autrement dit c'est comme si il ne pouvait y avoir de certitude que dans les régimes tyranniques tels que les monarchies absolues, les parlements totalitaires, les républiques sanglantes, les présidents despotiques, ou tout autre régime fort.

« Dans ce monde on ne peut pas avoir de certitude sur quoi que ce soit, excepté la mort et les taxes. » écrit Benjamin Franklin ${ }^{26}$ ». L'analyse des systèmes complexes va-t-elle lui donner raison?

L'analyse des gouvernances démocratiques des états du monde se confondent avec l'analyse des systèmes complexes. Ces gouvernements s'inscrivent dans de multiples contextes, en interactions les uns avec les autres ${ }^{27}$, qui interagissent avec les éléments (principes directeurs ${ }^{28}$ ) qui les composent tel que les règles implicites, explicites et mythique, leurs sous-systèmes et leurs membres parfois d'idéologies opposées. Toutes ces interactions forment leur structure qui évolue tantôt vers le changement ou vers l'homéostasie.

Leur analyse se place à un méta niveau.

Considérons la structure d'un système complexe (issue des interactions entre chacun de ses composants en interactions avec l'ensemble des contextes ${ }^{29}$ ). Cette structure est observée au temps $t 1$. Toute structure évolue en fonction des changements de contextes et des interactions avec les principes directeurs et de leurs interactions entre eux. Au temps t2 cette structure a subi des modifications. Certaines de ces modifications individuellement sont prévisibles mais la nouvelle structure t2 ne l'est pas. Elle a émergé de la totalité.

Il y a incertitude.

Comment vivre dans un état démocratique avec tout cela?

\footnotetext{
${ }^{26}$ Benjamin Franklin (1706-1790) signataire de la déclaration d'indépendance des Etats-Unis participe à la rédaction de la constituions des Etats-Unis est considéré comme faisant partie «des pères fondateurs des Etats-Unis »; il lutte contre l'esclavage.

${ }^{27}$ Andrée Piecq, Claude Lambert « The project: How to deal with coherence and incoherence”, UES-EUS Congress, 2014

${ }^{28}$ Andrée Piecq « De la Pensée Systémique à la Pratique de l'Organisation-Le « giroscope » p. 113 L'Harmattan

${ }^{29}$ Andrée Piecq, Claude Lambert "An essay of a systemic reading that can support a paradigm shift”, UES-EUS Seminar, 2013
} 
L'incertitude nous met devant l'inconnu, un inconnu présent dans tous les éléments et évènements de la vie. Vouloir vivre dans un monde de certitudes c'est comme si nous voulions vivre dans un monde homéostatique parfait ou tout est prévu et ou le changement n'existerai pas, comme dans des gouvernances absolues tyranniques.

Les Etats, dont la gouvernance est démocratique, sont des systèmes complexes, ne sont pas figés, ils vivent. Leurs contextes changent, ils s'inscrivent dans la mondialisation; changent au rythme des élections et des partis mis en place; leur structure change et ils vivent dans l'imprévisibilité de la forme émergeante qu'elle va prendre, ${ }^{30}$ ce qui a ce niveau est une liberté plutôt qu'une difficulté. En 2016, dans notre quotidien, pleins de peurs, d'angoisses, et de colères c'est comme si cette liberté portée par l'incertitude pouvait être un espoir pour l'avenir.

Nous concluons cet article comme nous l'avons commencé par la phrase de Sir Winston "Democracy is the worst form of government - except for all those other forms, that have been tried from time to time" que nous faisons nôtre après avoir observé un certain nombre de gouvernances de l'Europe et du monde à travers les siècles jusqu'à nos jours.

\section{Bibliographie}

Andrée Piecq 2011 «De la Pensée Systémique à la Pratique de l'Organisation-Le "giroscope » L'Harmattan

Andrée Piecq, Claude Lambert "An essay of a systemic reading that can support a paradigm shift" UES-EUS Seminar, 2013

Andrée Piecq, Claude Lambert "The project : How to deal with coherence and incoherence" UESEUS Congress, 2014

\footnotetext{
${ }^{30}$ Andrée Piecq, Claude Lambert "The project : How to deal with coherence and incoherence” UES-EUS Congress, 2014
} 
